\title{
Reliable Magnetic Resonance Imaging Based Grading System for Cervical Intervertebral Disc Degeneration
}

\author{
Lloydine J. Jacobs, Antonia F. Chen, James D. Kang, Joon Y. Lee \\ Department of Orthopaedic Surgery, University of Pittsburgh Medical Center, Pittsburgh, PA, USA
}

\begin{abstract}
Study Design: Observational.
Purpose: To develop a simple and comprehensive grading system for cervical discs that precisely, consistently and meaningfully presents radiologic and morphologic data.

Overview of Literature: The Thompson grading system is commonly used to classify the severity of degenerative lumbar discs on magnetic resonance imaging (MRI). Inherent differences in the morphological and physiological characteristics of cervical discs have hindered development of precise classification systems. Other grading systems have been developed for degenerating cervical discs, but their versatility and feasibility in the clinical setting is suboptimal.

Methods: MRIs of 46 human cervical discs were de-identified and displayed in PowerPoint format. Each slide depicted a single disc with a normal (grade 0 ) disc displayed in the top right corner for reference. The presentation was given to 25 physicians comprising attending spine surgeons, spine fellows, orthopaedic residents, and two attending musculoskeletal radiologists. The grading system included Grade 0 (normal height compared to C2-3, mid cleft still visible), grade 1 (dark disc, normal height), grade 2 (collapsed disc, few osteophytes), and grade 3 (collapsed disc, many osteophytes). The ease of use of the system was gauged in the participants and the interobserver reliability was calculated.

Results: The intraclass correlation coefficient for interobserver reliability was 0.87 , and 0.94 for intraobserver reliability, indicating excellent reliability. Ninety-five percent and 85 percent of the clinicians judged the grading system to be clinically feasible and useful in daily practice, respectively.

Conclusions: The grading system is easy to use, has excellent reliability, and can be used for precise and consistent clinician communication.
\end{abstract}

Keywords: Cervical spine; Degenerative disc disease; Grading or classification system; Magnetic resonance imaging

\section{Introduction}

Cervical disc degeneration is commonly associated with neck pain and radiculopathy in adults. It imposes a significant socioeconomic burden in terms of lost productivity, wages and increased health care expenditures [1]. Early detection and implementation of preventive measures can potentially delay the degenerative process and lessen the severity of the morbidity and socioeconomic burden $[1,2]$.

Magnetic resonance imaging (MRI) can identify disc

Received May 28, 2015; Revised Jul 4, 2015; Accepted Jul 5, 2015

Corresponding author: Lloydine J. Jacobs

Department of Orthopaedic Surgery, University of Pittsburgh Medical Center,

3471 FIfth Ave. LSK Building Suite 1011 Pittsburgh, PA 15213, USA

Tel: +1-646-533-9591 Fax: +1-412-605-3269, E-mail: Lloydine.Jacobs@gmail.com 
degeneration in the early stages and is the most accurate method used to assess disc integrity in the clinical setting. MRI visualizes disc hydration and morphology primarily based on proton density and water content. Normal intervertebral discs have a nucleus pulposus with high water and proteoglycan content that is depicted by a brighter signal on both T1- and T2-weighted MRI compared to the annulus and end plates. There is often a horizontal cleft of low signal intensity cells in the mid-substance of the nucleus pulposus in the middle of the nucleus pulposus. This is a normal finding in healthy and hydrated adult discs [3]. The fibers of the annulus fibrosus have a lower water and proteoglycan content, and emit a lower and darker signal.

Disc degeneration is characterized by a progressive loss of water and proteoglycan content that leads to a decrease in the T2-weighted signal of the nucleus pulposus, which attenuates the sharp distinction between the nucleus pulposus and the surrounding annulus fibrosus. As the degenerative process continues, the disc becomes increasingly more desiccated and begins to lose its height. In its final stages, the disc is severely compromised and osteophytes develop between the neighboring vertebral bodies secondary to changes in the normal biomechanical weight bearing forces.

Although most grading systems are subjective and susceptible to observer bias, standardized nomenclature is necessary to facilitate accurate and consistent comparison of data obtained from various investigations. The Thompson grading system is commonly used to classify the severity of degeneration of lumbar intervertebral discs based on MRI [4]. However, because of the inherent differences in the morphological and physiological characteristics of cervical discs compared to lumbar discs, more accurate systems are needed to aid in the description of intervertebral discs. Other grading systems have been developed for degenerating discs in the cervical spine [5-7], but their feasibility and reproducibility in the clinical setting is debatable.

A good classification system should allow for the presentation of data in an accurate manner to facilitate effective clinician communication so that appropriate and timely patient care is executed. The objective of this study is to demonstrate a simple, yet comprehensive grading system for cervical intervertebral discs capable of facilitating the presentation of radiologic and morphologic data in a precise, consistent, and meaningful manner.

\section{Materials and Methods}

Following approval by our Institutional Review Board (0306032), the cervical spine MRI data of the last 100 patients scanned at our outpatient facility were collected and de-identified. MRI data comprised an equal number of young (20-45 years of age), middle aged (45-65 years), and elderly ( $>65$ years) adults, and an equal number of males and females. Patients under 21 years of age, those with obvious non-degenerative pathology, and those with postsurgical changes were excluded.

De-identified MRI data of 46 human cervical discs were and presented in PowerPoint format on a CD. T1 weighted MRI data were used. Each slide depicted a single disc with a normal (grade 0) C2-3 disc displayed in the top right corner for reference. CDs containing the PowerPoint presentations were given to 10 attending orthopaedic spine surgeons, three fellows, 10 residents, and two musculoskeletal radiology attending physicians. The grading system comprised grade 0 (normal height compared to C2-3, with or without a cleft in the nucleus pulposus), grade 1 (dark disc, with normal height), grade 2 (collapsed disc, little or no osteophytes), grade 3 (collapsed disc, many osteophytes) (Fig. 1). The presentation was given three times in 4 week intervals. A short survey regarding ease of use was conducted.

As with most classification systems, the differentiation between grades 2 and 3 (minimal versus many osteophytes) is based on the clinician's impression. This further supports the need to evaluate the reliability of classification systems as well as their practicality in a busy clinical setting. Assessment of the reliability of our cervical disc degeneration classification system was determined using the intraclass correlation coefficient (ICC) for oneway random average measures using Predictive Analytics SoftWare Statistics ver. 20.0 (SPSS Inc., Chicago, IL, USA). ICC was calculated for both intraobserver and interobserver reliability; 0-0.2 indicated poor agreement, $0.3-0.4$ indicated fair agreement, $0.5-0.6$ indicated moderate agreement, $0.7-0.8$ indicated strong agreement, and $>0.8$ indicated excellent agreement. Clinician assessment of the classification system was reported in percentages.

\section{Results}

The average ICC for interobserver reliability was 0.87 (average range, 0.81-0.93) and 0.94 (average range, 0.9-0.98) 
Grade 0: Normal, light grey center, mid cleft still visible

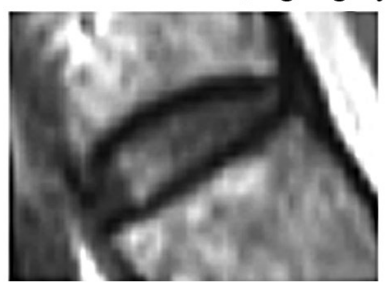

Grade 1: Dark disc, not collapsed

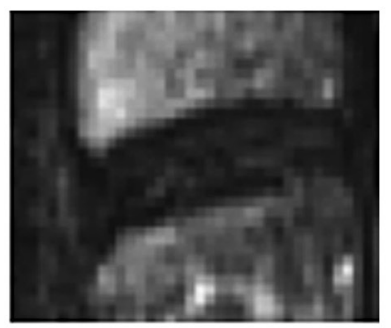

Grade 2: Dark disc, coooapsed with minimal osteophytes

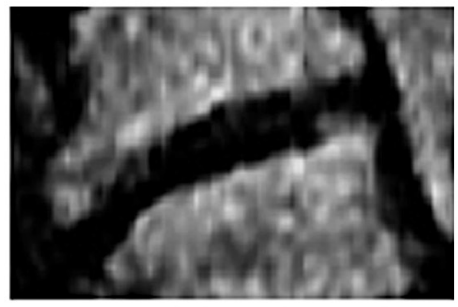

Grade 3: Dark disc, collapsed with many osteophytes

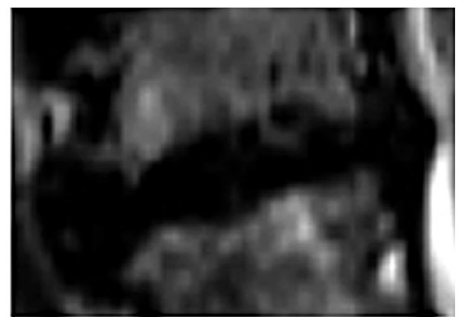

Fig. 1. Grading System for cervical disc degeneration.

for intraobserver reliability, which indicated excellent reliability for both. Ninety-five percent of the clinicians involved reported that this grading system was easy to use. Eighty-five percent indicated that they would begin using this grading system in their clinical practice.

\section{Discussion}

A good classification system allows for the presentation of data in an accurate manner to facilitate effective clinician communication so that appropriate and timely patient care is provided. Many lumbar intervertebral disc grading systems are commonly used for grading discs throughout the spine. However, because cervical discs have inherent differences in morphology and function, grading systems specifically designed for cervical discs are required to facilitate clear and accurate communication.

While most grading systems are based on lumbar intervertebral discs, published systems have been specifically developed for cervical discs. The feasibility and reproducibility in the clinical setting is debatable. The objective of this study, therefore, was to demonstrate a simple, yet comprehensive grading system for cervical intervertebral discs capable of facilitating the presentation of radiologic and morphologic data for clinical and research purposes.

The first objective grading system was developed to investigate the effect of disc degeneration on flexibility [8]. The system was based on changes in the disc height compared to adjacent discs, extent of osteophyte formation, and presence of endplate sclerosis. The numerical scale ranged from 0 (normal) to very severe ( $<25 \%$ disc height). Each disc was given a score based on the sum of points from each category with grade 1 being 0 to 1.5 points and grade 4 exceeding 6 points. The goal of this system was to avoid the use of subjective terms like "mild", "moderate", or severe". The system proved cumbersome and impractical in a busy clinical practice.

Pfirrmann et al. [9] developed a classification system based on Thompson's system for lumbar intervertebral disc degeneration. He reported substantial-to-excellent intraobserver and interobserver agreement. Sorensen proposed a grading system for lumbar disc and muscle degeneration using low-field MRI and reported fair to moderate interobserver agreement and almost perfect intraobserver agreement [10]. Christe et al. [5] used MRI to assess cervical disc degeneration by looking at four features (osteophytes and/or disc narrowing, disc prolapse, annular tears, and decrease in signal intensity). The author's claim of good reliability has not yet been substantiated.

Although grading systems for intervertebral discs have been proposed for years, more recent attention has been placed on measuring and increasing the reproducibility of these systems. The ICC or the Kappa ( $\mathrm{k}$ ) value is acceptable for measuring both the inter- and intraobserver reliabilities. A $\mathrm{k}$-value of $<0.00$ is interpreted as no agreement, 0.01 to 0.20 as slight agreement, 0.21 to 0.40 as fair agreement, 0.41 to 0.60 as moderate agreement, 0.61 to 0.80 as substantial agreement, and $>0.81$ as almost perfect agreement [11].

A grading system for cervical discs based on the inten- 
sity of the nucleus pulposus signal, degree of disc prolapse, and adjacent vertebral bone marrow signal has been developed [6]. The system includes 12 possible grades (grades A through L). The $\mathrm{K}$-value for the interobserver reliability of their grading system was reportedly 0.45 (moderate agreement), but it is unlikely to be a practical clinical tool for routine use.

Another proposed classification system for cervical intervertebral discs has a specific focus on improving both the reliability and feasibility of a new grading system for routine clinical use. The system is based on the signal intensity and structure of the nucleus pulposus, the distinction between the nucleus and the annulus fibrosus, and the height of the disc. The reported $\mathrm{k}$-values for intraobserver and interobserver reliability averaged 0.93 and 0.78 , respectively [7]. The system is less demanding than others previously proposed in the literature, but is still quite involved.

The classification system presented in this paper is objective, comprehensive, simple to use, and reproducible. It is based on the biological progression of disc degeneration. The normal healthy disc is well hydrated with a clear distinction between the nucleus pulposus and the annulus fibrosis on MRI (grade 0: normal height compared to C23 , with or without a cleft in the nucleus pulposus). As the disc becomes progressively more desiccated, loss of heterogeneity between the nucleus pulposus and the annulus fibrosus occurs, which gives the disc a homogenously dark appearance (grade 1: dark disc, with normal height). As desiccation continues, the disc begins to lose its ability to withstand compressive forces and begins to lose its height (grade 2: collapsed disc, little or no osteophytes). This is followed by the progressive development of osteophytes secondary to the change in the normal biomechanical contact forces between adjacent vertebrae, with many osteophytes indicating longstanding degenerative changes (grade 3: collapsed disc, many osteophytes).

This classification system demonstrated an ICC of 0.87 and 0.94 for interobserver reliability and intraobserver reliability, respectively. Both indicated excellent reliability. Ninety-five percent of the clinicians who participated in the study felt that this grading system was simple and easy to use in a busy clinical practice. Eighty-five percent indicated that they would start to use this grading system in their practice. These results are the highest values that we could find in the literature.

There are a few limitations of this study. Only 46 discs were used and were presented in the form of a PowerPoint presentation on a CD. Twenty-five blinded physicians participated in the grading process and the presentation was given only three times. This does exceed what is usually found in the literature, but larger numbers with more frequent trials could have strengthened our findings. Lastly, the presentation was distributed on CDs, so the resolution of the images displayed could vary depending on the computer it was viewed on.

\section{Conclusions}

The presented grading system is easy to use, has excellent interobserver reliability, and can be used to facilitate communication between medical professionals in a precise, consistent, and meaningful manner.

\section{Conflict of Interest}

No potential conflict of interest relevant to this article was reported.

\section{References}

1. Hogg-Johnson S, van der Velde G, Carroll LJ, et al. The burden and determinants of neck pain in the general population: results of the Bone and Joint Decade 2000-2010 Task Force on Neck Pain and Its Associated Disorders. Spine (Phila Pa 1976) 2008;33 (4 Suppl):S39-51.

2. Jacobs LJ, Vo N, Kang JD. Identifying inflammatory targets for biologic therapies for spine pain. PM $\mathrm{R}$ 2011;3:S12-7.

3. Aguila LA, Piraino DW, Modic MT, Dudley AW, Duchesneau PM, Weinstein MA. The intranuclear cleft of the intervertebral disk: magnetic resonance imaging. Radiology 1985;155:155-8.

4. Thompson JP, Pearce RH, Ho B. Correlation of gross morphology and chemical composition with magnetic resonance images of human lumbar intervertebral discs. Trans Orthop Res Soc 1988;13:276.

5. Christe A, Laubli R, Guzman R, et al. Degeneration of the cervical disc: histology compared with radiography and magnetic resonance imaging. Neuroradiology 2005;47:721-9.

6. Kolstad F, Myhr G, Kvistad KA, Nygaard OP, Leivseth G. Degeneration and height of cervical discs 
classified from MRI compared with precise height measurements from radiographs. Eur J Radiol 2005;55:415-20.

7. Miyazaki M, Hong SW, Yoon SH, Morishita Y, Wang JC. Reliability of a magnetic resonance imagingbased grading system for cervical intervertebral disc degeneration. J Spinal Disord Tech 2008;21:288-92.

8. Mimura M, Panjabi MM, Oxland TR, Crisco JJ, Yamamoto I, Vasavada A. Disc degeneration affects the multidirectional flexibility of the lumbar spine. Spine (Phila Pa 1976) 1994;19:1371-80.
9. Pfirrmann CW, Metzdorf A, Zanetti M, Hodler J, Boos N. Magnetic resonance classification of lumbar intervertebral disc degeneration. Spine (Phila $\mathrm{Pa}$ 1976) 2001;26:1873-8.

10. Solgaard Sorensen J, Kjaer P, Jensen ST, Andersen P. Low-field magnetic resonance imaging of the lumbar spine: reliability of qualitative evaluation of disc and muscle parameters. Acta Radiol 2006;47:947-53.

11. Landis JR, Koch GG. The measurement of observer agreement for categorical data. Biometrics 1977;33:159-74. 